\title{
Desempenho de clones de seringueira de origem amazônica no planalto do Estado de São Paulo(1)
}

\author{
Paulo de Souza Gonçalves(2), Nelson Bortoletto(3), Rosana Sambugaro(4), Edson Luiz Furtado(5), \\ Ondino Cleante Bataglia( ${ }^{(6)}$, Altino Aldo Ortolani( ${ }^{(7)}$ e Gentil Godoy Júnior ${ }^{(8)}$
}

Resumo - O objetivo deste trabalho foi avaliar a adaptabilidade e expressão fenotípica de caracteres superiores de dez clones amazônicos de seringueira (Hevea spp.) no planalto do Estado de São Paulo em um período de 10 anos, obedecendo ao delineamento de blocos ao acaso com três repetições e parcelas lineares de seis plantas. O clone IAN 3156 foi o mais produtivo, com média de $65,57 \mathrm{~g}$ de

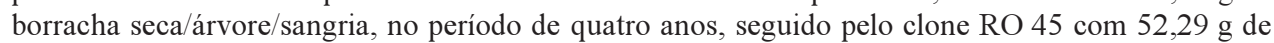
borracha seca/árvore/sangria, enquanto o clone-testemunha, RRIM 600, produziu 41,04 g/árvore/sangria. Todos os clones apresentaram crescimento vigoroso. O perímetro do caule na abertura do painel variou de $37,01 \mathrm{~cm}$ (IAN 3193) a 49,41 cm (IAN 4493). A porcentagem de plantas aptas à sangria variou de 30,0\% (IAN 3703) a 93,75\% (IAN 6323). Exceto os clones IAN 3156 e IAN 4493 com $7,00 \mathrm{~mm}$ e $6,32 \mathrm{~mm}$, respectivamente, todos os outros clones apresentaram espessura de casca virgem inferior ao clone RRIM 600, que apresentou $6,18 \mathrm{~mm}$. O clone IAN 3193 apresentou maior incidência de quebra do ponteiro pelo vento. Todos os clones estudados apresentaram baixa incidência de secamento de painel. O bom desempenho dos clones IAN 3156, RO 45, Fx 3899 e IAN 4493 permite que possam ser experimentados em larga escala, envolvendo diferentes ambientes no Estado de São Paulo.

Termos para indexação: casca, espessura, secamento, sangria, antracnose, rendimento

\section{Performance of Amazonian rubber tree clones in the plateau region of the State of São Paulo, Brazil}

Abstract - The objective of this paper was to evaluate, for a period of 10 years, the adaptability and phenotypic expression of superior characters of 10 clones of rubber tree (Hevea spp.) grown in the plateau of São Paulo State, Brazil. The clones in the field were arranged in a randomized block design with three replicates and six plants per linear plots. The results showed that the clone IAN 3156 recorded highest yield (65.57 g/tree/tap) followed by RO 45 (52.29 g/tree/tap), whereas the control clone RRIM 600 recorded $41.04 \mathrm{~g} /$ tree/tap over four years of tapping. All selected clones were vigorous, with girth range at opening varying from $37.01 \mathrm{~cm}$ (IAN 3193) to $49.41 \mathrm{~cm}$ (IAN 4493). The percentage range of plants able for opening were $30.0 \%$ for IAN 3703 to $93.75 \%$ for IAN 6323. Except for IAN 3156 with $7.0 \mathrm{~mm}$ and for IAN 4493 with $6.32 \mathrm{~mm}$ other clones had low thick virgin bark at open compared to the control clone RRIM 600, which recorded $6.18 \mathrm{~mm}$. Concerned to wind damage, except for IAN 3193 all the studied clones experienced low incidence of wind damage. All clones showed low to very low incidence of tree dryness. A complementary evaluation of the best clones (IAN 3156 , RO 45, Fx 3899 and IAN 4493) in different environment of the São Paulo State is suggested, to evaluate besides yielding other secondary characters with the purpose of future recommendation of planting material in large scale.

Index terms: bark, thickness, drying, tapping, anthracnosis, yields.

\footnotetext{
(1) Aceito para publicação em 2 de março de 2001. Parcialmente financiado pela Fapesp.

(2)Embrapa/Instituto Agronômico, Programa Integrado de São Paulo, Programa Seringueira do Centro Café e Plantas Tropicais (CCTP) do Instituto Agronômico (IAC), Caixa Postal 28 , CEP 13001-970 Campinas, SP. E-mail: paulog@cec.iac.br

(3)IAC, Núcleo de Agronomia do Noroeste, Centro de Ação Regional (CAR), Caixa Postal 401, CEP 15500-000 Votuporanga, SP. E-mail: nan.iac@votuporanga.com.br

(4)Programa IAC/CCTP. Bolsista da Fapesp. E-mail: rosambugaro@hotmail.com
}

(5)Universidade Estadual Paulista, Fac. de Ciências Agronômicas, Dep. de Defesa Fitossanitária, Caixa Postal 237, CEP 18603-970 Botucatu, SP. E-mail: elfurtado@fca.unesp.br

(6)IAC, Centro de Solos e Recursos Agroambientais, Caixa Postal 28, CEP 13001-970 Campinas, SP. E-mail: ondino@barao.iac.br

(7)IAC, Centro de Ecofisiologia e Biofísica, Seção de Climatologia Agrícola, Caixa Postal 28, CEP 13001-970 Campinas, SP E-mail: altino@cec.iac.br

(8) IAC, Estação Experimental de Agronomia de Ubatuba, Caixa Postal 59, CEP 11680-000 Ubatuba, SP. E-mail: iac-uba@iconet.com.br 


\section{Introdução}

Um dos principais fatores limitantes na recomendação de clones de seringueira é o longo período de testes de campo ( 25 a 30 anos), que compreende desde a polinização controlada até a fase de teste, após a qual são recomendados aos produtores para plantio em larga escala.

Tentando contornar esse problema, os institutos de pesquisa têm experimentado clones elites, originados de outras regiões, em solos e clima prevalecentes de áreas heveícolas da região a que se destina o material. Tratando-se de clones amazônicos, a busca de áreas que propiciem o escape da seringueira ao mal-das-folhas causada pelo fungo Microcyclus ulei (P. Henn) v. Arx, vem se constituindo como uma excelente alternativa para a utilização desses clones.

No decorrer dos últimos anos, o Instituto Agronômico tem-se preocupado em buscar informações quanto ao comportamento de clones amazônicos sob condições ambientais que possibilitem evitar o M. ulei.

Segundo Ho (1979), o tipo de solo pode realçar ou restringir o potencial de um clone, ao passo que outros fatores ecológicos, tais como o vento e a propensão à incidência de doenças que, quando severas, podem reduzir seriamente o referido potencial.

Em relação ao desempenho de clones amazônicos, existem poucos dados que permitam recomendálos para o seu plantio na região do planalto do Estado de São Paulo. A maioria das informações sobre eles foram obtidas na região de origem (Pinheiro et al., 1980; Gonçalves et al., 1982; Paiva et al., 1983; Alves et al., 1988), no Sul da Bahia (Dunham et al., 1983; Gomes et al., 1983; Marques et al., 1988) e no litoral do Estado de São Paulo (Gonçalves et al., 1991).

O objetivo deste trabalho foi estudar a expressão fenotípica dos caracteres superiores de clones amazônicos de seringueira, e sua adaptabilidade ao planalto do Estado de São Paulo.

\section{Material e Métodos}

O experimento foi instalado em janeiro de 1989 , no Núcleo de Agronomia do Noroeste do Instituto Agronômico (IAC), no Município de Votuporanga a 20²0' S de latitude, $49^{\circ} 58^{\prime} \mathrm{W}$ de longitude e $510 \mathrm{~m}$ de altitude, em solo Podzólico Vermelho-Escuro, latossólico eutrófico a moderado, textura arenosa média.

O clima predominante na região é oAw (Köppen), com estação seca definida, temperatura média anual de $22^{\circ} \mathrm{C}$, intervalo médio de $18,4^{\circ} \mathrm{C}$ a $23,9^{\circ} \mathrm{C}$, umidade relativa média anual em torno de $70,0 \%$, com extremos de $77,1 \%$ em fevereiro e $59,0 \%$ em agosto. A pluviosidade média anual é de $1.344 \mathrm{~mm}$, com $74 \%$ das chuvas ocorridas de outubro a março e $26 \%$ de abril a setembro.

Foram utilizados dez clones: oito, resultantes de cruzamentos feitos no antigo Instituto Agronômico do Norte Fx 985, Fx 3899, IAN 3156, IAN 3193, IAN 3703, IAN 4493, IAN 6323 e IAN 6721, mais o RO 45, clone primário, resultado de coleta efetuada em 1974 na região do Jaru, Estado de Rondônia (Viégas \& Gonçalves, 1974), e o clone malaio RRIM 600 (testemunha), o mais plantado no Estado de São Paulo. Os parentais são apresentados na Tabela 1.

O delineamento estatístico adotado foi o de blocos ao acaso, com três repetições, parcelas lineares de seis plantas para cada clone. O espaçamento entre linhas foi de $7,0 \mathrm{~m}$, e entre plantas, 3,0 m. Os clones amazônicos foram recebidos da Embrapa-Centro de Pesquisa Agroflorestal da Amazônia (CPAA), mediante introduções efetuadas na coleção de clones da antiga Seção de Plantas Tropicais do IAC, no período de 1980 a 1983, situada no Núcleo Experimental de Campinas (NEC). Dois anos antes da instalação do experimento, os clones foram multiplicados em jardim clonal, e selecionados de acordo com seu vigor, e, em seguida, recepados, objetivando a produção de hastes rejuvenescidas. Passados 18 meses da recepagem, as hastes produzidas foram enxertadas por método convencional, pelo qual as gemas maduras dos clones foram enxertadas em porta enxertos com 12 a 14 meses de idade, oriundos de sementes de polinização aberta de uma população de pés francos plantados na Estação de Agronomia de Pindorama, SP.

Os clones foram avaliados anualmente; no primeiro ano, o perímetro do caule foi medido a uma altura de $0,50 \mathrm{~m}$ acima do calo de enxertia; a partir do segundo ano, a 1,20 m de altura. Dados de produção foram registrados somente a partir do sétimo ano de idade, nas plantas da parcela que apresentavam perímetro de caule superior a $45 \mathrm{~cm}$.

O sistema utilizado para avaliar a produção de borracha no primeiro ano foi o $1 / 2 \mathrm{~S} \mathrm{~d} / 25 \mathrm{~d} / 7.11 \mathrm{~m} / \mathrm{y}$ - sangria em meia-espiral $(1 / 2 \mathrm{~S})$, realizada em intervalos de dois dias $(\mathrm{d} / 2)$; esta atividade foi realizada em cinco dias da semana ( $5 \mathrm{~d} / 7)$, durante 11 meses do ano ( $11 \mathrm{~m} / \mathrm{y})$. O sistema utilizado no segundo ano foi o $1 / 2 \mathrm{~S} \mathrm{~d} / 35 \mathrm{~d} / 7.11 \mathrm{~m} / \mathrm{y}$. 
ET $2,5 \% \mathrm{~Pa} 10 / \mathrm{y}$ - sangria em meia espiral $(1 / 2 \mathrm{~S})$, realizada em intervalos de três dias (d/3); a atividade foi realizada em cinco dias da semana ( $5 \mathrm{~d} / 7)$, durante 11 meses do ano $(11 \mathrm{~m} / \mathrm{y})$; a sangria foi estimulada com $2,5 \%$ do ingrediente ativo de ethefon (ET 2,5\%), aplicado por pincelamento no painel de sangria $(\mathrm{Pa})$, dez vezes por ano $(10 / y)$; o sistema utilizado no terceiro e quarto ano foi $1 / 2 \mathrm{~S} d / 45 \mathrm{~d} / 7.11 \mathrm{~m} / \mathrm{y}$. ET 5,0\% Pa 8/y - sangria em meia espiral $(1 / 2 \mathrm{~S})$, realizada a cada quatro dias de intervalo $(\mathrm{d} / 4)$, em cinco dias da semana ( $5 \mathrm{~d} / 7)$, durante 11 meses do ano $(11 \mathrm{~m} / \mathrm{y})$; neste caso, a estimulação foi feita com ethefon a $5,0 \%$ do ingrediente ativo (ET 5,0\%), aplicado por pincelamento no painel $(\mathrm{Pa})$, oito vezes ao ano $(8 / \mathrm{y})$

A produção de borracha foi determinada em todas as árvores das parcelas mediante a pesagem do látex coagulado, secado em condições normais de sombra e ventilação, e coletado em tigelas, duas vezes ao mês, ao longo do período de avaliação de cada árvore. O total de borracha coletada no ano, por árvore, foi dividido pelo número de "coágulos"; o resultado foi expresso em gramas/árvore/sangria. A média geral do total de árvores/clone foi expressa em gramas/árvore/clone/ano. Desta maneira, foi possível estimar a produção por clone/hectare/ano, segundo recomendações da Embrapa (1989). No primeiro ano, a coleta foi feita em 240 árvores; no segundo, em 340 árvores; no terceiro, em 380 árvores, e no quarto ano, em 400 árvores, o que correspondeu a $60 \%, 85 \%, 95 \%$ e $100 \%$ de um estande de 400 plantas por hectare. Foram consideradas, no sistema, $\mathrm{d} / 2, \mathrm{~d} / 3$ e d/4, 140, 100 e 72 sangrias/ano, respectivamente (Virgens Filho et al., 1999).

Foram tomadas amostras de casca virgem para determinação da espessura e número de anéis de vasos laticíferos a 1,0 $\mathrm{m}$ de altura da base do caule de cada árvore, com o auxílio de um "vazador". A espessura da casca foi determinada por meio de paquímetro; o número de anéis de vasos laticíferos foi obtido por meio de cortes longitudinais da casca.

A incidência, no clone, da antracnose do painel, causada pelo fungo Colletotrichum gloeosporioides (Penz.) Saac., foi feita em todas as plantas. O grau de severidade foi medido conforme a intensidade de ataques da doença nos painéis, mediante uma escala de notas graduais, de 1 a 5 ( 1 = altamente resistente; $2=$ resistente; $3=$ moderadamente resistente; $4=$ suscetível; $5=$ altamente suscetível).

Ao longo da condução do experimento, foram utilizadas práticas culturais convencionais ao cultivo da seringueira preconizadas por Cardoso (1982)

\section{Resultados e Discussão}

Na Tabela 2 são apresentados os resultados das análises de variância de cada indivíduo, referentes aos quatro anos de produção. Em virtude da utilização de diferentes sistemas de sangria em cada ano, a análise agrupada não foi realizada.

Considerando-se que cerca de 240 árvores/ha estavam aptas à sangria no primeiro ano, observou-se que o clone IAN 3156 diferiu estatisticamente dos demais a $5 \%$ de probabilidade, com $40,97 \mathrm{~g}$, e os clones Fx 3899, RRIM 600 e Fx 985 apresentaram a segunda maior produção de borracha seca, com $18,63,18,26$ e 17,86 gramas/sangria/ano, respectivamente (Tabela 3 ).

O uso do estimulante ethefon a 2,5\% (sistema de sangria $1 / 2 \mathrm{~S} \mathrm{~d} / 35 \mathrm{~d} / 7.11 \mathrm{~m} / \mathrm{y}$. ET $2,5 \% \mathrm{~Pa} 10 / \mathrm{y}$ ), no segundo ano, proporcionou aumento de produção em todos os clones (Tabela 3). O clone IAN 3156 foi o

Tabela 1. Parentais dos clones amazônicos selecionados no Núcleo de Agronomia do Noroeste do Instituto Agronômico (IAC). Votuporanga, $\mathrm{SP}^{(1)}$

\begin{tabular}{|c|c|}
\hline Clone & Parentais \\
\hline IAN 3156 & Fx 516 (F $4552 \times$ AVROS 363) x PB 86 \\
\hline IAN 3193 & Fx 516 (F $4542 \times$ AVROS 363) x PB 86 \\
\hline IAN 3703 & Fx 4371 (F 4542 x PB 86) x PB 86 \\
\hline IAN 4493 & Fx 4421 (F 4573 x PB 86) x Tjir 1 \\
\hline IAN 6323 & Tjir 1 x Fx 3810 (F 4542 x AVROS 363) \\
\hline IAN 6721 & Fx 43-655 [Fx 213 (F $4542 \times$ AVROS 183) x AVROS 183] x PB 86] \\
\hline Fx 985 & F 315 x AVROS 183 \\
\hline Fx 3899 & F $4542 \times$ AVROS 363 \\
\hline $\mathrm{RO} 45$ & Clone primário \\
\hline RRIM 600 & Tjir 1 x PB 86 \\
\hline
\end{tabular}

(1)IAN: Instituto Agronômico do Norte; F: Ford (clone primário); Fx: Cruzamento Ford; AVROS: Algemene Vereniging Rubber planters Oostkust Sumatra; Tjir: Tjirandji; PB: Prang Besar; RO: Rondônia; RRIM: Rubber Reseach Institute of Malaysia. 
mais produtivo nesse ano, com $66,61 \mathrm{~g}$, seguido pelo clone RO 45, com 59,84 g/s/a; estes foram os únicos superiores ao clone RRIM 600 (48,38 g/s/a), porém não diferiram estatisticamente entre si a $5 \%$ de probabilidade.

A aplicação de ethefon a 5\% no terceiro e quarto ano propiciou aumento de produção na maioria dos clones. Os clones IAN 3156 e RO 45 foram os mais produtivos no terceiro ano, com $89,13 \mathrm{~g} / \mathrm{s} / \mathrm{a}$, $82,12 \mathrm{~g} / \mathrm{s} / \mathrm{a}$, respectivamente, e no quarto ano, com $91,99 \mathrm{~g} / \mathrm{s} / \mathrm{a}, 69,62 \mathrm{~g} / \mathrm{s} / \mathrm{a}$, respectivamente. Essas produções foram superiores às do primeiro ano em 117\%, $451 \%$ e $125 \%, 367 \%$ respectivamente, porém não diferiram estatisticamente entre si a 5\% de probabilidade. Por outro lado, o clone Fx 985 apresentou redução na sua produção com a aplicação de ethefon 5\%. Segundo Jonge (1955), Levandowsky (1961) e Abraham et al. (1976), a resposta ao percentual de aplicação de estimulante pode variar entre clones ou até entre indivíduos de um mesmo clone.

A produção média anual dos clones IAN 3156 , RO 45 e IAN 4493, nos quatro primeiros anos, foi de $2.499,1.940$ e $1.519 \mathrm{~kg} / \mathrm{ha} /$ ano de borracha seca, superior em $80 \%, 40 \%$ e $10 \%$, respectivamente, em relação ao clone RRIM 600 (Tabela 3). Todos os outros apresentaram médias iguais ou inferiores à testemunha (RRIM 600). O clone IAN 3156, em Votuporanga, apresentou a maior média de produção nos quatro primeiros anos, como também uma boa média de crescimento em perímetro do caule ao longo dos 11 anos de avaliação (Tabela 4). O seu bom comportamento em áreas de escape foi também comprovado em Açailândia (MA), com média de produção de 42,31 g de borracha seca por sangria, no primeiro ano, e média do perímetro do caule em torno de $66 \mathrm{~cm}$, aos 11 anos de idade (Pinheiro et al., 1980).

A produção de borracha seca anual dos clones IAN 3156, RO 45, IAN 4493, e Fx 3899, foi superior na média dos quatro anos, em relação ao clonetestemunha RRIM 600 (Tabela 3).

Nos 11 anos, o aumento do perímetro variou de clone para clone. Com exceção dos clones Fx 3899 e da testemunha (RRIM 600), os clones mais produtivos, IAN 3152, RO 45, Fx 3899 e IAN 4493, alcançaram média de perímetro do caule superior a $45 \mathrm{~cm}$ no sétimo ano, idade de abertura do painel (Tabela 4).

As diferenças entre os anos de crescimento do perímetro do caule foram estatisticamente significativas a1\% de probabilidade, o que indica variabilidade de incremento entre os anos (Tabela 5). Entre clones não foram observadas variações significativas em relação a essa variável.

O maior incremento médio anual no período de imaturidade foi apresentado pelo clone IAN 4493, com 7,06 cm, seguido pelos clones IAN 3156 , IAN 6323, RO 45 e IAN 6721, com médias $6,96 \mathrm{~cm}$, $6,89 \mathrm{~cm}, 6,77 \mathrm{~cm}$ e $6,71 \mathrm{~cm}$ respectivamente; superiores ao incremento do clone-testemunha (RRIM 600), com 5,61 cm (Tabela 6). Ao longo dos onze anos, as maiores médias de incremento entre os clones foram observadas do terceiro para o quarto ano de desenvolvimento, com valor de $7,76 \mathrm{~cm}$ de

Tabela 2. Valores dos quadrados médios, coeficientes de variação (CV\%) e média geral da produção, em gramas de borracha seca por sangria por árvore, referentes à análise de variância dos 10 clones de seringueira do Experimento de Avaliação em Pequena Escala instalado no Núcleo de Agronomia do Noroeste do Instituto Agronômico (IAC). Votuporanga, SP.

\begin{tabular}{lccccc}
\hline Fonte de & G.L. & \multicolumn{4}{c}{ Quadrados médios $^{(1)}$} \\
\cline { 3 - 6 } variação & & Ano 1 & Ano 2 & Ano 3 & Ano 4 \\
\hline Blocos & 2 & $16,7167^{\text {ns }}$ & $39,7719^{\text {ns }}$ & $163,3502^{\text {ns }}$ & $201,8070^{\text {ns }}$ \\
Clones & 9 & $254,5403^{* *}$ & $459,5651^{* *}$ & $1155,3499^{* *}$ & $892,5606^{* *}$ \\
Residuo & 18 & 6,2050 & 86,5388 & 134,6484 & 159,9022 \\
\hline CV\% & & 15,964 & 24,272 & 23,209 & 24,275 \\
Média & & 15,60 & 38,33 & 49,99 & 52,09 \\
\hline
\end{tabular}

(1) Ano 1: sistema de sangria $1 / 2 \mathrm{~S} \mathrm{~d} / 25 \mathrm{~d} / 7.11 \mathrm{~m} / \mathrm{y}$; Ano 2: sistema de sangria $1 / 2 \mathrm{~S} \mathrm{~d} / 3$ 5d/7.11m/y. ET 2,5\% Pa 10/y; Ano 3: sistema de

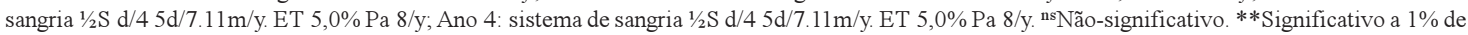
probabilidade, pelo teste $\mathrm{F}$. 
Tabela 3. Estimativas de produção em gramas de borracha seca por sangria por árvore e em quilogramas de borracha seca por hectare por ano e porcentagem de ganho em relação à testemunha (RRIM 600), referentes aos quatro anos de sangria de 10 clones selecionados no Experimento de Avaliação em Pequena Escala no Núcleo de Agronomia do Noroeste do Instituto Agronômico. Votuporanga, SP(1)

\begin{tabular}{|c|c|c|c|c|c|c|c|c|c|c|c|}
\hline \multirow[t]{2}{*}{ Clones } & \multicolumn{2}{|c|}{ Ano 1} & \multicolumn{2}{|c|}{ Ano 2} & \multicolumn{2}{|c|}{ Ano 3} & \multicolumn{2}{|c|}{ Ano 4} & \multicolumn{2}{|c|}{ Média } & \multirow{2}{*}{$\begin{array}{c}\text { Produção } \\
\text { relativa } \\
(\%)\end{array}$} \\
\hline & $\mathrm{g} / \mathrm{s} / \mathrm{a}$ & $\mathrm{kg} / \mathrm{ha} / \mathrm{ano}$ & $\mathrm{g} / \mathrm{s} / \mathrm{a}$ & $\mathrm{kg} / \mathrm{ha} / \mathrm{ano}$ & $\mathrm{g} / \mathrm{s} / \mathrm{a}$ & $\mathrm{kg} / \mathrm{ha} / \mathrm{ano}$ & $g / s / a$ & $\mathrm{~kg} / \mathrm{ha} / \mathrm{ano}$ & $\mathrm{g} / \mathrm{s} / \mathrm{a}$ & $\mathrm{kg} / \mathrm{ha} / \mathrm{ano}$ & \\
\hline IAN 3156 & $40,97 \mathbf{a}$ & 1,376 & $66,61 a$ & 2.355 & $89,13 a$ & 2.439 & 91,99 & 3.827 & 72,18 & 2.499 & 180 \\
\hline $\mathrm{RO} 45$ & $14,92 \mathrm{bc}$ & 501 & $59,84 \mathrm{a}$ & 2.116 & $82,12 \mathrm{a}$ & 2.247 & 69,62 & 2.896 & 56,63 & 1.940 & 140 \\
\hline Fx 3899 & $18,63 \mathrm{~b}$ & 626 & $39,52 \mathrm{ab}$ & 1.397 & $53,74 \mathrm{ab}$ & 1.470 & 49,84 & 2.073 & 40,43 & 1.392 & 100 \\
\hline IAN 4493 & $13,57 \mathrm{bcd}$ & 455 & $39,42 \mathrm{ab}$ & 1.397 & $59,33 \mathrm{ab}$ & 1.623 & 62,55 & 2.602 & 43,72 & 1.519 & 110 \\
\hline Fx 985 & $17,86 \mathrm{~b}$ & 600 & $40,08 \mathrm{ab}$ & 1.417 & $37,02 \mathrm{~b}$ & 1.013 & 36,99 & 1.538 & 32,99 & 1.142 & 82 \\
\hline IAN 873 & - & - & $29,22 \mathrm{~b}$ & 1.033 & $35,42 b$ & 969 & 33,78 & 1.405 & 32,81 & 1.136 & 82 \\
\hline IAN 6323 & $9,37 \mathrm{~d}$ & 314 & $27,18 b$ & 961 & $37,11 \mathrm{~b}$ & 1.015 & 36,91 & 1.935 & 27,64 & 1.056 & 76 \\
\hline IAN 6721 & $7,03 \mathrm{~d}$ & 236 & $24,94 b$ & 882 & $38,42 \mathrm{~b}$ & 1.051 & 42,20 & 1.755 & 28,15 & 981 & 71 \\
\hline IAN 3193 & $11,36 \mathrm{bcd}$ & 382 & $27,46 \mathrm{~b}$ & 971 & $29,59 b$ & 810 & 49,13 & 2.044 & 29,39 & 1.052 & 76 \\
\hline RRIM 600 & $18,26 \mathrm{~b}$ & 614 & $48,38 \mathrm{ab}$ & 1.711 & $56,48 \mathrm{ab}$ & 1.545 & 53,76 & 1.677 & 44,22 & 1.387 & 100 \\
\hline
\end{tabular}

(1)Médias seguidas da mesma letra, na coluna, não diferem entre si a 5\% de probabilidade pelo teste de Tukey; Ano 1: sangria no sistema 1/2.S d/2 5d/7.11m/y: 140 sangrias/ano, 240 árvores/ha; Ano 2: sangria no sistema ${ }^{2} / 2 \mathrm{~S} \mathrm{~d} / 35 \mathrm{~d} / 7.11 \mathrm{~m} / \mathrm{y}$. ET 2,5\% Pa 10/y: 100 sangrias/ano, 340 árvores/ha; Ano 3: sangria no sistema $1 / 2 \mathrm{~S} \mathrm{~d} / 45 \mathrm{~d} / 7.11 \mathrm{~m} / \mathrm{y}$. ET 5,0\% Pa 8/y: 72 sangrias/ano, 380 árvores/ha; Ano 4: sistema de sangria no sistema $1 / 2 \mathrm{~S} \mathrm{~d} / 3$ 5d/7.11 $\mathrm{m} / \mathrm{y}$. ET 2,5\% Pa 10/y: 100 sangrias/ano, 340
$1 / 2 \mathrm{~S} \mathrm{~d} / 4$ 5d/7.11m/y. ET 5,0\% Pa 8/y: 72 sangrias/ano, 400 árvores/ha.

Tabela 4. Perímetro médio anual do caule $(\mathrm{cm})$, referente a seleção de clones amazônicos e a testemunha RRIM 600, instalados no Núcleo deAgronomia do Noroeste do Instituto Agronômico (IAC). Votuporanga, SP.

\begin{tabular}{|c|c|c|c|c|c|c|c|c|c|c|c|c|}
\hline \multirow[t]{2}{*}{ Clone } & \multirow{2}{*}{$\begin{array}{l}\mathrm{N}^{0} \mathrm{de} \\
\text { árvores }\end{array}$} & \multicolumn{7}{|c|}{ Período de imaturidade ${ }^{(1)}$} & \multicolumn{4}{|c|}{ Período adulto $^{(2)}$} \\
\hline & & Ano 1 & Ano 2 & Ano 3 & Ano 4 & Ano 5 & Ano 6 & Ano 7 & Ano 8 & Ano 9 & Ano 10 & Ano 11 \\
\hline IAN 3156 & 14 & 7.15 & 10,81 & 18,05 & 29,35 & 37,42 & 44,29 & 48,69 & 51,10 & 58,67 & 62.50 & 67,30 \\
\hline IAN 3193 & 7 & 6,79 & 7,97 & 13,68 & 18,86 & 24,64 & 31,25 & 37,01 & 42,42 & 44,50 & 56,50 & 63,75 \\
\hline IAN 3703 & 13 & 6,84 & 8,92 & 13,01 & 19,87 & 27,84 & 33,16 & 40,56 & 40,73 & 49,14 & 54,27 & 59,21 \\
\hline IAN 4493 & 17 & 7,58 & 10,58 & 18,39 & 28,50 & 35,56 & 42,21 & 49,41 & 50,58 & 52,11 & 54,27 & 69,05 \\
\hline IAN 6323 & 16 & 7,67 & 11,94 & 19,78 & 29,20 & 37,16 & 43,42 & 48,20 & 51,36 & 57,78 & 61,19 & 64,81 \\
\hline IAN 6721 & 12 & 6,97 & 11,01 & 18,25 & 25,73 & 32,00 & 41,50 & 46,95 & 49,23 & 55,95 & 57,98 & 67,77 \\
\hline Fx 985 & 13 & 5,90 & 6,49 & 10,38 & 17,08 & 24,06 & 30,23 & 38,57 & 40,40 & 47,59 & 57,14 & 62,96 \\
\hline Fx 3899 & 12 & 6,54 & 7,42 & 17,06 & 23,02 & 25,45 & 32,45 & 40,60 & 41,62 & 48,82 & 53,79 & 56,35 \\
\hline RO 45 & 18 & 8,48 & 12,69 & 19,22 & 26,88 & 35,70 & 42,06 & 47,37 & 47,67 & 58,50 & 60,60 & 66,71 \\
\hline RRIM 600 & 60 & 5,39 & 9,42 & 10,64 & 17,53 & 26,61 & 33,34 & 39,28 & 44,24 & 51,06 & 54,10 & 64,57 \\
\hline Média & & $6,93+0,88$ & $9,73+2,02$ & $15,85+3,58$ & $23,60+4,94$ & $30,64+5,48$ & $37,39+5,71$ & $43,66+4,85$ & $45,93+4,51$ & $52,41+5,04$ & $57,23+3,25$ & $64,29+3,76$ \\
\hline
\end{tabular}

(1)Ano 1: dados coletados a 0,50 m acima do calo de enxertia; do ano 2 ao ano 6; dados coletados a 1,20 m do calo de enxertia; ano 7: ocorreu abertura do painel. (2)Período adulto: avaliação após abertura do painel. 
perímetro. Exceto no primeiro e décimo anos, as médias do incremento dos 10 clones não diferiram estatisticamente entre si a $5 \%$ de probabilidade, pelo teste de Tukey.

$\mathrm{Na}$ fase adulta, o clone IAN 3193 apresentou o perímetro com maior média anual $(6,69 \mathrm{~cm})$ seguido dos clones RRIM 600, Fx 985 e IAN 6721, com valores de 6,32, 6,10 e $5,21 \mathrm{~cm}$, respectivamente. $\mathrm{Na}$ fase imatura, a média geral do crescimento do perímetro dos clones foi de $6,24 \mathrm{~cm}$, e, na fase adulta, de $5,15 \mathrm{~cm}$, ou seja, ocorreu uma diminuição no crescimento em perímetro após a abertura do painel de $1,09 \mathrm{~cm}$. A sangria geralmente contribui para a redução do crescimento do perímetro nas árvores de um mesmo clone; porém, existem clones de alta capacidade de produção e vigor que não apresentam redução do crescimento do perímetro durante o período de avaliação (Abraham \& Tayler, 1967). Após a abertura do painel, o fotossintetizado é repartido em duas fontes competidoras: látex explotado, e crescimento, em diâmetro, do caule. Em alguns clones, a maior parte do fotossintetizado é direcionada para o látex explotado, reduzindo o crescimento (Wycherley, 1976). O desenvolvimento dos clones no período de explotação é um caráter muito importante na manutenção da constância da produção, e que, por sua vez, contribuirá na redução da quebra de árvores pelo

Tabela 5. Valores dos quadrados médios da análise de variância, coeficientes de variação $(\mathrm{CV} \%)$ e média geral do incremento do perímetro do caule $(\mathrm{cm})$, referente ao período de 11 anos de avaliação de 10 clones do experimento de avaliação em pequena escala instalado no Núcleo de Agronomia do Noroeste do Instituto Agronômico (IAC). Votuporanga, SP

\begin{tabular}{lcc}
\hline Fonte de variação & G.L. & Incremento do caule \\
\hline Blocos & 2 & $1,24^{\text {ns }}$ \\
Clones & 9 & $3,83^{* *}$ \\
Resíduo (clones) & 18 & 1,60 \\
Anos & 10 & $103,05^{* *}$ \\
Clones x anos & 90 & $9,03^{* *}$ \\
Anos x blocos & 20 & $4,30^{\text {ns }}$ \\
Resíduo (anos) & 180 & 4,30 \\
\hline CV\% (clones) & & 6,46 \\
CV\% (anos) & & 35,15 \\
Média & & 5,90 \\
\hline
\end{tabular}

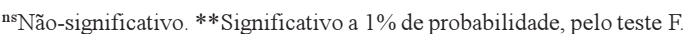

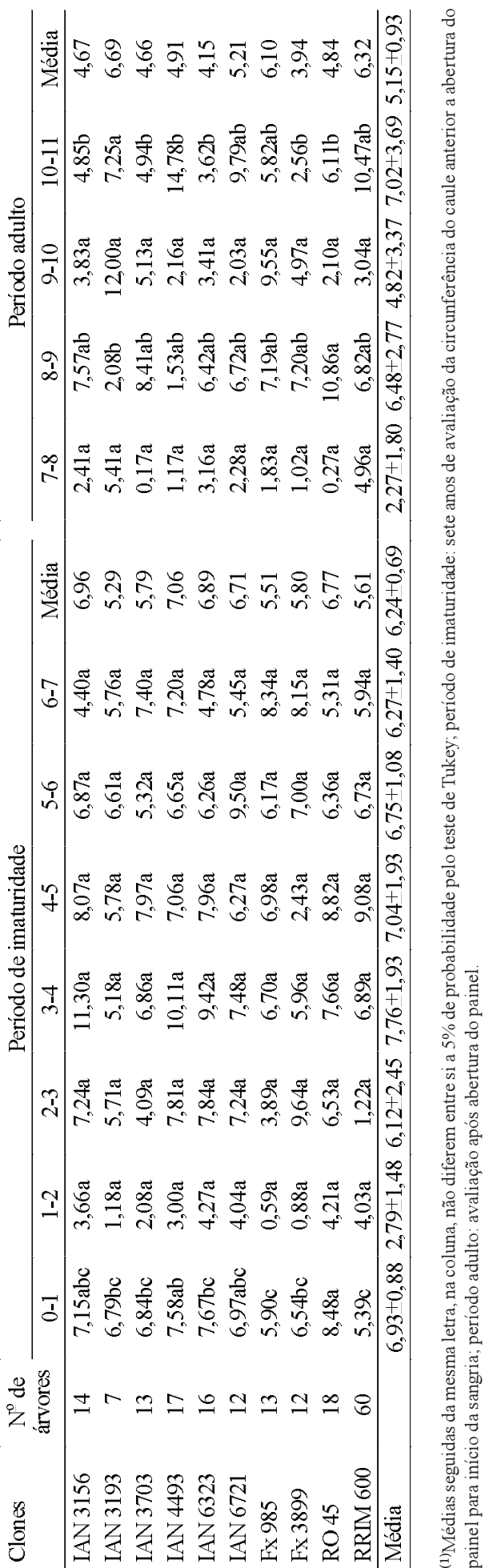


vento. Em geral, os incrementos em perímetro são significativamente menores na maturidade (fase adulta) em comparação com o da fase de imaturidade, o que indica que o crescimento do caule das árvores nesses clones é afetado pela explotação de látex (Gonçalves et al., 1993).

A porcentagem de árvores/clone aptas a sangria variou de $30,00 \%$ (IAN 3703) a 93,75\% (IAN 6323), e apenas $20 \%$ dos clones em estudo mostraram um porcentual de árvores aptas à sangria superior ao RRIM 600 (83,00\%) (Tabela 7). A espessura de casca virgem aos sete anos de idade variou desde $5,10 \mathrm{~mm}$ (IAN 3703 ) até 7,00 mm (IAN 3156). Os clones IAN $3156(7,00 \mathrm{~mm})$ e IAN $4493(6,32 \mathrm{~mm})$ apresentaram médias de espessura de casca superiores às do clone RRIM 600 (6,18 mm). A espessura de casca é importante, pois influi indiretamente na produção do clone e facilita a prática da sangria (Gonçalves et al., 1994). O clone IAN 3156 apresentou o maior número de anéis de vasos laticíferos $(38,20)$, seguido dos clones IAN $4493(32,00)$ e RO 45 (31,18). Os demais clones apresentaram número de anéis de vasos laticíferos inferiores ao do clone RRIM 600 (29,06). Segundo Webster \&
Paardekooper (1989), o número de anéis é o fator mais importante do sistema laticífero, pois está significativamente correlacionado com a produção.

O clone IAN 3156 apresentou maior espessura da casca, maior número de anéis de vasos laticíferos, e um dos maiores perímetros do caule na abertura do painel. Porém, mostrou certa propensão ao secamento do painel (50\%); trata-se de uma enfermidade fisiológica das seringueiras que aumenta com a intensidade da sangria (Sharples \& Lambourne, 1924; Bealing \& Chua, 1972). Também a estimulação do painel pode causar ou aumentar a gravidade do mal. Isto é particularmente evidente em clones propensos a essa doença (Ho et al., 1974). Segundo Sanderson \& Sutcliffe (1921) e Rhodes (1930), o consumo de casca durante a sangria também parece ser um fator importante, pois, esses pesquisadores constataram baixo consumo de casca quando a incidência da doença era alta.

A incidência de quebra do caule pelo vento variou entre $0,0 \%$ e $20,0 \%$ (Tabela 7 ). O clone IAN 3193 apresentou a maior incidência (20\%), seguido pelos clones IAN 6721 (15,0\%) e RRIM 600 (10,0\%). Os clones IAN 3156, IAN 3703, Fx 985,

Tabela 7. Dados médios do perímetro do caule, número de plantas aptas à sangria, espessura de casca média, número de anéis de vasos laticíferos, árvores com seca de painel, árvores quebradas pelo vento e a susceptibilidade à antracnose, relativos à abertura do painel, relacionados à seleção de clones amazônicos e à testemunha RRIM 600 no Núcleo de Agronomia do Noroeste do Instituto Agronômico (IAC). Votuporanga, SP.

\begin{tabular}{|c|c|c|c|c|c|c|c|}
\hline \multirow[t]{2}{*}{ Clones } & \multicolumn{4}{|c|}{ Abertura do painel $^{(1)}$} & \multirow{2}{*}{$\begin{array}{l}\text { Árvores } \\
\text { com seca } \\
\text { de painel } \\
(\%)\end{array}$} & \multirow{2}{*}{$\begin{array}{c}\text { Árvores } \\
\text { quebradas } \\
\text { pelo vento } \\
(\%)\end{array}$} & \multirow{2}{*}{$\begin{array}{c}\text { Susceptibilidade } \\
\text { à antracnose } \\
\text { do painel }{ }^{(2)}\end{array}$} \\
\hline & $\begin{array}{l}\text { Perímetro } \\
\text { do caule } \\
\text { (cm) }\end{array}$ & $\begin{array}{c}\text { Árvores } \\
\text { aptas à } \\
\text { sangria }(\%)\end{array}$ & $\begin{array}{c}\text { Espessura } \\
\text { da casca } \\
(\mathrm{mm})\end{array}$ & $\begin{array}{c}\mathrm{N}^{\circ} \text { de anéis } \\
\text { de vasos } \\
\text { laticíferos }\end{array}$ & & & \\
\hline IAN 3156 & 48,69 & 55,00 & 7,00 & 38,20 & 5,0 & 5,0 & 1 \\
\hline IAN 3193 & 37,01 & 50,00 & 5,20 & - & 0,0 & 20,0 & 1 \\
\hline IAN 3703 & 40,56 & 30,00 & 5,10 & - & 0,0 & 5,0 & 3 \\
\hline IAN 4493 & 49,41 & 88,25 & 6,32 & 32,00 & 5,0 & 0,0 & 1 \\
\hline IAN 6323 & 48,20 & 93,75 & 5,18 & 19,23 & 5,0 & 0,0 & 1 \\
\hline IAN 6721 & 46,95 & 75,00 & 5,47 & 27,43 & 0,0 & 15,0 & 2 \\
\hline Fx 985 & 38,57 & 46,00 & 5,15 & 25,00 & 10,0 & 5,0 & 1 \\
\hline Fx 3899 & 40,60 & 34,00 & 5,33 & 25,00 & 5,0 & 5,0 & 1 \\
\hline $\mathrm{RO} 45$ & 47,37 & 72,22 & 5,24 & 31,18 & 10,0 & 5,0 & 1 \\
\hline RRIM 600 & 39,28 & 83,00 & 6,18 & 29,06 & 6,0 & 10,0 & 3 \\
\hline Média & $43,66 \pm 4,85$ & $62,72+22,76$ & $5,62 \pm 0,65$ & $28,39+5,67$ & $4,60 \pm 3,72$ & $7,00 \pm 6,32$ & \\
\hline
\end{tabular}

(1)A abertura do painel foi realizada no sétimo ano de avaliação, nas árvores que alcançaram $45 \mathrm{~cm}$ do perímetro do caule, tomados a 1,20 m de altura. (2) Notas de avaliação: 1 = altamente resistente; 2 = resistente; 3 = moderadamente resistente; 4 = suscetível; 5 = altamente suscetível 
Fx 3899 e RO 45 mostraram índices de 5,0\%, e nos clones IAN 4493 e IAN 6323 esse índice foi nulo. Segundo Ho et al. (1974), a quebra do caule pelo vento, entre outros fatores, pode ser causada pelo baixo crescimento do caule na fase adulta.

Quanto à resistência à antracnose do painel, causada por Colletotrichum gloeosporioides, destacaram-se como altamente resistentes os clones: IAN 3156, IAN 4493, IAN 6323, IAN 3193, Fx 985, Fx 3899 e RO 45; como resistente, IAN 6721, e moderadamente resistentes, IAN 3703 e o RRIM 600, utilizado, este, como testemunha (Tabela 7).

\section{Conclusões}

1. Na abertura do painel, os clones IAN 4493, IAN 3156 e IAN 6323 apresentam o maior perímetro de caule.

2. Na abertura do painel, os clones IAN 3156 e IAN 4493 apresentam maior espessura de casca virgem e maior número de anéis de vasos laticíferos.

3. O clone IAN 3156 possui maior propensão à seca de painel de sangria quando se utiliza ethefon a $5,0 \%$.

4. Os clones IAN 3193 e IAN 6721 mostram maior propensão à quebra pelo vento.

5. Os clones IAN 3156 e RO 45 apresentam melhor desempenho médio de produção nos três primeiros anos de sangria.

6. Os clones IAN 3156, IAN 4493, IAN 6323, IAN 3193, Fx 985, Fx 3899 e RO 45 apresentam alta resistência à antracnose do painel.

\section{Agradecimentos}

Às laboratoristas Andréa Cardoso Guerreiro e Lígia Regina Lima Gouvêa, pelas análises do sistema laticífero contido na casca da seringueira; aos técnicos agrícolas Ari de Camargo e Isaac Jesus de Souza, do Núcleo Experimental de Campinas e Estação Experimental de Votuporanga, respectivamente, pelo manejo aplicado ao experimento durante a fase de desenvolvimento do experimento.

\section{Referências}

ABRAHAM, P. D.; P'NG, T. C.; LEE, C. K.; SIVAKUMARAN, S.; MANIKAM, B.; YEAH, C. P.
Ethrel stimulation of Hevea. In: INTERNATIONAL RUBBER CONFERENCE, 1975, Kuala Lumpur. Proceedings... Kuala Lumpur: Rubber Research Institute of Malaysia, 1976. p. 347-350.

ABRAHAM, P. D.; TAYLER, R. S. Tapping of Hevea brasiliensis. Tropical Agriculture, St. Augustine, v. 44, n. 1, p. 1-11, 1967.

ALVES, R. M.; PINHEIRO, F. S. V.; GOMES, V. A.; LOPES, P. R. de A. Blocos monoclonais de TracuateuaPA. In: FACULDADE DE CIÊNCIAS AGRÁRIAS DO PARÁ. Relatório bienal: 1986/87. Belém: Convênio Embrapa/FCAP-Seringueira, 1988. p. 53-58.

BEALING, F. J.; CHUA, S. E. Output, composition and metabolic activity of Hevea latex in relation to tapping intensity and the onset of brown blast. Journal of the Rubber Research Institute of Malaysia, Kuala Lumpur, v. 23 , n. 3, p. 204-231, 1972

CARDOSO, M. Instruções para a cultura da seringueira. Campinas: Instituto Agronômico, 1982. 43 p. (Boletim, 196)

DUNHAM, R. J.; SILVA, E. R. da; SANTOS,A. G. Comportamento dos clones de seringueira e novos materiais recomendados para futuros plantios na Fazenda Três Pancadas-Ituberá e Camamu-BA. In: SEMINÁRIO BRASILEIRO SOBRE RECOMENDAÇÕES DE CLONES DE SERINGUEIRA, 1., 1982, Brasília. Anais... Brasília: Sudhevea/Embrapa/Embrater, 1983. p 65-87.

EMBRAPA. Centro Nacional de Pesquisa de Seringueira e Dendê (Manaus, AM). Melhoramento genético da seringueira. Manaus, 1989. 23 p. (Documentos, 10).

GOMES,A. R. S.; VIRGENS FILHO,A. C.; MARQUES, J. R. B.; SANTOS, P. M. Avaliação de clones de seringueira (Hevea spp.) no sul da Bahia. In: SEMINÁRIO BRASILEIRO SOBRE RECOMENDAÇÕES DE CLONES DE SERINGUEIRA, 1., 1982, Brasília. Anais... Brasília: Sudhevea, 1983. p. 139-158.

GONÇALVES, P. de S.; CARDOSO, M.; CAMPANA, M.; FURTADO, E. L.; TANZINI, M. R. Desempenho de novos clones de seringueira da série IAC. Seleções promissoras para a região do planalto do Estado de São Paulo. Pesquisa Agropecuária Brasileira, Brasília, v. 29, n. 8, p 1215-1224, ago. 1994.

GONÇALVES, P. de S.; CARDOSO, M.; LAVORENTI, C.; BOAVENTURA, M. A. M.; SAES, L. A. Comportamento de alguns clones de seringueira em Registro no Vale do Ribeira, SP. Campinas: Instituto Agronômico, 1991. 26 p. (Boletim Científico, 23). 
GONÇALVES, P. de S.; CARDOSO, M.; MENTE, E. M.; MARTINS, A. L. M.; GOTTARDI, M. V. C. Desempenho preliminar de clones de seringueira na região de São José do Rio Preto, planalto do Estado de São Paulo. Bragantia, Campinas, v. 52, n. 2, p. 119-130, 1993

GONÇALVES, P. de S.; PAIVA, J. R. de; TRINDADE, D. R.; VALOIS, A. C. C.; VIEGAS, I. de J. M. Comportamento preliminar de alguns clones de seringueira. Pesquisa Agropecuária Brasileira, Brasília, v. 17, n. 10, p. 1447-1456, out. 1982.

HO, C. Y. Contributions to improve the effectiveness of breeding, selection and planting recommendations of Hevea brasiliensis Muell. Arg. Ghent: Faculty of Agricultural Sciences, 1979. 341 p. Ph.D. Thesis.

HO, C. Y; CHAN, H. Y.; LIM, T. M. Enviromax planting recommendations: a new concept in choice of clones. In RUBBER RESEARCH INSTITUTE OF MALAYSIA PLANTER'S CONFERENCE, 1974, Kuala Lumpur. Proceedings... Kuala Lumpur: Rubber Research Institute of Malaysia, 1974. p. 293-320

JONGE, P. de. Stimulation in Hevea brasiliensis. Further observations on the effects of yield stimulants. Journal of the Rubber Research Institute of Malaysia, Kuala Lumpur, v. 14, p. 383-405, 1955.

LEVANDOWSKY, D. W. Clonal variations in reaction of Hevea brasiliensis to stimulation of latex yield and to bark regeneration. In: NATURAL RUBBER RESEARCH CONFERENCE, 1961, Kuala Lumpur. Proceedings.. Kuala Lumpur: Rubber Research Institute of Malaysia, 1961. p. $270-281$

MARQUES, J. R. B.; GOMES, A. R. S.; VIRGENS FILHO, A. C.; SANTOS, P. M. Informações sobre germoplasma de seringueira (Hevea spp.) na Estação Experimental Djalma Bahia. Itabuna: Centro de Pesquisas do Cacau, 1988. 24 p. (Boletim Técnico, 157).

PAIVA, J. R. de; GONÇALVES, P. de S.; VALOIS, A. C. C. Avaliação preliminar do comportamento de no- vos clones de seringueira (Hevea spp.) em Manaus (AM). Pesquisa Agropecuária Brasileira, Brasília, v. 18, n. 2, p. 148-158, fev. 1983

PINHEIRO, E.; PINHEIRO, F. S. V.; ALVES, R. M. Comportamento de alguns clones de Hevea, em Açailândia, na região pré-amazônica maranhense: dados preliminares. In SEMINÁRIO NACIONAL DA SERINGUEIRA, 3., 1980 Manaus. Anais... Brasília: SUDHEVEA, 1980. p. 1011-1129.

RHODES, E. Brown blast: some considerations as to its nature. Journal of the Rubber Research Institute of Malaysia, Kuala Lumpur, v. 2, n. 1, p. 1-5, 1930.

SANDERSON, A. R.; SUTCLIFFE, H. Brown blast: an investigation into its causes and methods of treatment London: Rubber Growers Association, 1921. 98 p.

SHARPLES, A.; LAMBOURNE, J. Field experiments relating to brown blast disease of Hevea brasiliensis. Malaysian Agricultural Journal, Kuala Lumpur, v. 12, p. 290-296, 1924.

VIÉGAS, I. de J. M.; GONÇALVES, P. de S. Terceira coleta de material nativo de alta produção em seringais do Estado do Acre e Território Federal de Rondônia: relatório. Belém: Instituto de Pesquisa Agropecuária do Norte, 1974. $46 \mathrm{p}$

VIRGENS FILHO, A. de C.; MATOS, C. R.; REIS, E. L.; SCOMPARIN, C. H. J. Cultivo de seringueira para produção de borracha natural. Viçosa, MG: Centro de Produção Técnicas, 1999. 64 p.

WEBSTER, C. C.; PAARDEKOOPER, E. C. The botany of the rubber tree. In: WEBSTER, C. C.; BAULKWILL, W. J. (Ed.). Rubber. New York: Longman, 1989. p. $57-84$

WYCHERLEY, P. R. Tapping and partition. Journal of the Rubber Research Institute of Malaysia, Kuala Lumpur, v. 24, p. 169-194, 1976 